BULL. AUSTRAL. MATH. SOC.

$04 A 20,03 E 05$

VOL. 31 (1985), 475-477.

\title{
INTERSECTION PROPERTIES OF \\ FUNCTIONS ON CARDINALS
}

\author{
GREG G. GIBBON
}

This thesis consists of three parts, each one concerned with a problem in Combinatorial Set Theory.

Part 1 deals with problems involving set mappings of unrestricted order. It is well-known that set mappings of order less than $k$ always have a free set of size $K$. We prove an inversion theorem which allows us to apply existing results about set mappings of order $\kappa$ to get results about set mappings of unrestricted order. See [3].

Part 2 considers generalizations of the regressive functions studied by Fodor in his classical paper [2]. Fodor showed that if a function $f: \kappa \rightarrow k$ satisfies $f(\alpha)<\alpha$ on a set that is stationary in $k$, then $f$ is constant on a stationary subset of this set. We develop conditions that guarantee that there exists a stationary constant set for a function $f: k \rightarrow[k]^{\leq \lambda}$ which satisfies $f(\alpha) \subseteq \alpha$ on some subset $S$ of $k$. We also consider the stationary subsets of the set $[\rho]^{<K}$.

Part 3 is the main part of the thesis. We study two generalizations of property $B$. A family of sets $A$ has property $B$ if there is a set $T$ such that $A \cap T \neq \emptyset$ and yet $A \notin T$ for all sets $A$ in $A$. These two requirements may be restated in terms of the characteristic functions of the sets in $A$ as follows: $\exists x\left(x_{T}(x)=x_{A}(x)=1\right)$ and

Received 13 February 1985. Thesis submitted to University of Queensland September 1984. Degree approved February 1985. Supervisor: Dr N.H. Williams.

Copyright Clearance Centre, Inc. Serial-fee code: 0004-9727/85 $\$ A 2.00+0.00$. 
$\exists y\left(X_{T}(y) \neq X_{A}(y)=1\right)$. We study properties $P$ and $R$ which are statements about families of functions and these are motivated by this alternative characterization of property $B$.

We say that a family of functions $A=\left\{\phi_{\alpha}: \kappa+\theta ; \alpha<\rho\right\}$ possesses property $P$ if there is a function $T$ into $\theta$ such that for all $\phi$ in $A$ and every value $\beta<\theta$ we have $\exists x(T(x)=\phi(x)=\beta)$ and $\exists y(T(y) \neq \phi(y)=B)$ true. We say that $A$ possesses property $R$ if for each $\phi$ in $A$ there exists a value $\beta<\theta$ such that $\exists x(T(x)=\phi(x)=\beta)$ and $\exists y(T(y) \neq \phi(y)=B)$ holds.

A classical theorem of Bernstein says that for any infinite cardinal $\kappa$, a family of $K$ sets each of size $K$ always possesses property $B$. One may then ask what conditions would ensure a positive result if we strengthen the requirement $A \notin T$ to $|A \cap T|<\mu$ for a suitable cardinal $\mu$. A large body of work was done on this property, denoted by $B(\mu)$, by Erdös and Hajnal in 1961 (see [1]). This amounts to adding a transversal property onto the requirements for property $B$. We generalize this transversal property to families of functions by requiring that for each value $\beta<\theta$ and each $\phi$ we have $\left|T^{-1}(\beta) \cap \phi^{-1}(\beta)\right|<\mu$, and so we get properties $P(\mu)$ and $R(\mu)$.

Erdös and Hajnal introduced certain intersection conditions which were imposed on families of sets in order to guarantee that the family possesses property $B(\mu)$. We investigate a generalization of these intersection conditions to families of functions. The results in some cases resemble those for families of sets. However, there are cases where the positive results hold only for functions into large cardinals.

We conclude with a study of families of functions that are more restricted than those treated previously. Those families previously treated were generalized from the families of sets studied in conjunction with property $B$, and the intersection conditions were also direct generalizations of those developed for sets. In the context of families of functions, however, these classical intersection conditions are actually counterproductive for certain cases and we introduce new intersection conditions that guarantee positive results for the same properties $P, R$, and so on. This analysis requires new methods and the results do not 
resemble those of the previous chapter.

\section{References}

[1] P. Erdös and A. Hajnal, "On a property of families of sets", Acta Math. Acad. Sci. Hungar. 12 (1961), 87-123.

[2] G. Fodor, "Eine Bemerkung zur Theorie der Regressiven Funktionen", Acta Sci. Math. Szeged 17 (1956), 139-143.

[3] Greg. G. Gibbon, "Set mappings of unrestricted order", Bull. Austral. Math. Soc. 28 (1983), 199-206.

18 Haig Road,

Milton,

Queensland 4064,

Australia. 